\title{
AVALIAÇÃO DOS COMPOSTOS BIOATIVOS PRESENTES NO RESÍDUO DE GOIABA VERMELHA (Psidium guajava $L$.) DESIDRATADA
}

\author{
F. P. MORAES ${ }^{1}$, E. S. da SILVA ${ }^{1}$, P. M. ROCHA ${ }^{1}$, A. S. da SILVA ${ }^{1}$ e R. T. P. \\ CORREIA $^{1}$ \\ ${ }^{1}$ Universidade Federal do Rio Grande do Norte, Departamento de Engenharia Química. \\ Campus Universitário, Natal, RN, Brasil. 59072-970. \\ E-mail para contato: franciscapereira.moraes@gmail.com
}

RESUMO - O Brasil possui grandes indústrias processadoras de goiaba (Psidium guajava L.), as quais geram volumes consideráveis de resíduos agroindustriais. Dessa forma, o objetivo desse trabalho foi avaliar o teor bioativo desse resíduo, através da determinação do teor de compostos fenólicos totais (método de Folin-Ciocalteu), ácido ascórbico (titulométrico), carotenoides (método espectrofotométrico) e atividade antioxidante (radical DPPH). A secagem foi conduzida em secador convectivo a temperatura de $70^{\circ} \mathrm{C}$ e velocidade do ar 5 $\mathrm{m} / \mathrm{s}$. Foram observados valores expressivos de ácido ascórbico $(47,58 \mathrm{mg} / 100 \mathrm{~g})$ e carotenoides $(262,01 \mu \mathrm{g} / 100 \mathrm{~g})$ no resíduo seco. O material também apresentou quantidade relevante de compostos fenólicos $(133,73 \mathrm{mg} \mathrm{GAE} / 100 \mathrm{~g})$ e boa atividade antioxidante $(9,06$ $\mu \mathrm{mol} \mathrm{TE} / \mathrm{g}$ ). Os resultados mostram a presença de importantes fitoquímicos no resíduo desidratado da goiaba vermelha, sendo ele um potencial ingrediente para a indústria de alimentos.

\section{INTRODUÇÃO}

Na economia do país, o agronegócio brasileiro apresenta participação expressiva no produto interno bruto (PIB) (CEPEA, 2014). A agroindústria pode trabalhar estrategicamente para o desenvolvimento sustentável de sistemas de produção de frutas tropicais nativas e exóticas (RAMOS, 2008). Apesar disso, por volta de 50\% do total da matéria-prima é descartada durante o processamento na forma de folhas, talos, cascas e sementes, sendo normalmente utilizado como matéria-prima para compostagem ou encaminhado para a alimentação animal (TOLENTINO E SILVA, 2008).

A goiabeira é uma das árvores mais conhecidas do Brasil e é encontrada em qualquer região brasileira pela facilidade com que suas sementes são dispersas por pássaros e pequenos animais. A colheita da fruta goiaba (Psidium guajava L., Figura 1), excelente fonte de vitamina $C$, pode ser feita entre abril e junho e de novembro a fevereiro. Suas sementes são pequenas e numerosas (BRASIL, 2002).

Estudos com o resíduo da goiaba oriundo da agroindústria têm sido realizados na tentativa de utilizá-lo como subprodutos na indústria alimentícia (ROBERTO, 2012). O interesse por essa matéria-prima se justifica pelos experimentos previamente realizados que apontaram o resíduo da goiaba como fonte alternativa de antioxidantes naturais (NASCIMENTO, 2010) com significantes concentrações de compostos fenólicos totais (SOUSA et al., 2011b). 
Assim, o presente trabalho tem como objetivo mostrar o potencial bioativo do resíduo da goiaba vermelha desidratado em secador de bandejas. Para isso, são apresentadas as concentrações de compostos fenólicos totais, atividade antioxidante, ácido ascórbico e carotenoides totais presentes no resíduo obtido como subproduto do processamento da polpa. Os resultados pretendem revelar o real valor bioativo desse resíduo, abundantemente encontrado no Brasil.

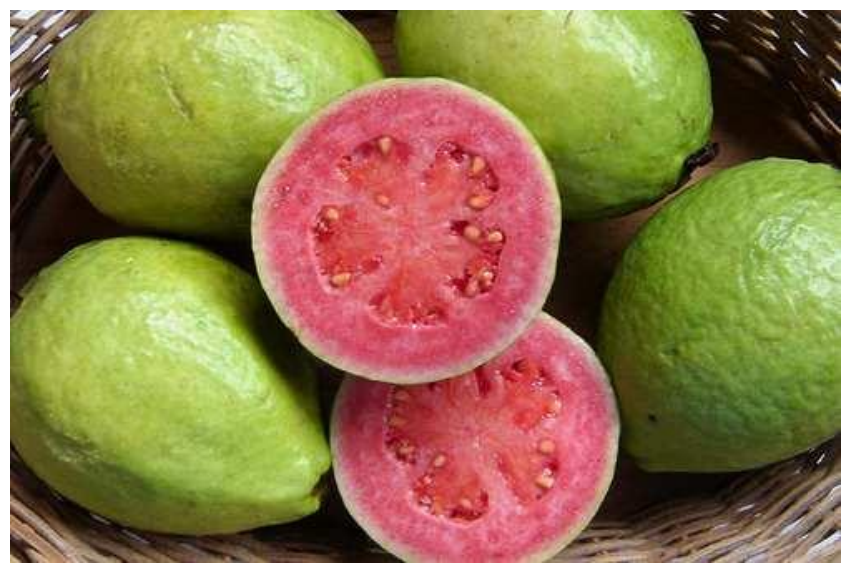

Figura 1 - A goiaba vermelha. Fonte: CULTURA DA GOIABA (2014).

\section{MATERIAIS E MÉTODOS}

O resíduo da goiaba vermelha foi cedido pela indústria produtora de polpa de fruta congelada Nordeste Fruit, localizada na cidade do Natal/RN. O resíduo foi submetido à secagem convectiva a $70{ }^{\circ} \mathrm{C}$ em secador convectivo de bandejas com velocidade do ar no secador de $5 \mathrm{~m} / \mathrm{s}$ e duração da secagem de 510 minutos. Em seguida, os resíduos desidratados foram moídos em moinho multi-uso (Tecnal, Brasil). O pó obtido foi posteriormente utilizado para o preparo dos extratos aquosos para as determinações de fenólicos totais e atividade antioxidante ou utilização direta para determinação de carotenoides e ácido ascórbico (Figura 2).

\section{Obtenção dos extratos}

Os extratos aquosos foram obtidos pelo método de extração não sequencial. Foi pesado $1 \mathrm{~g}$ do pó, o qual foi homogeneizado em $50 \mathrm{~mL}$ de água destilada sob agitação por 1 hora à temperatura ambiente $\left(25^{\circ} \mathrm{C}\right)$. Após o período de agitação, a solução foi filtrada a vácuo, usando papel de filtro Whatman $n^{\circ} 1$ e centrifugada a $3600 \mathrm{rpm}$ por 10 minutos. Em seguida, o sobrenadante foi separado e envolvido com papel alumínio para evitar degradação pela luz (Figura 2). 


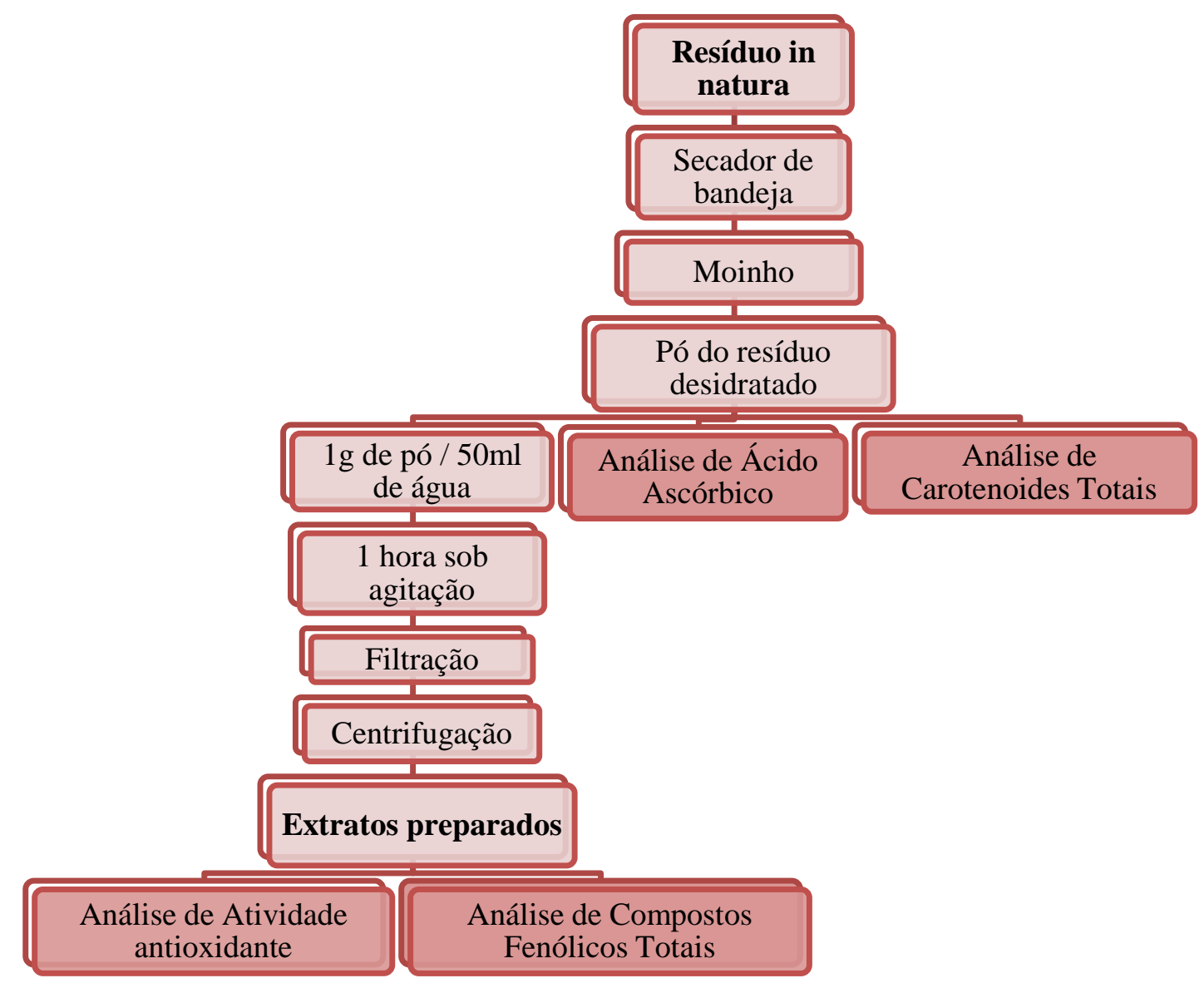

Figura 2 - Sequência de procedimentos de obtenção dos extratos para a realização das análises funcionais.

\section{Determinação dos compostos fenólicos totais (CFT)}

A determinação foi realizada conforme Correia et al. (2004). Uma alíquota de $1 \mathrm{~mL}$ do extrato foi transferida para tubos de ensaio e recebeu os reagentes na sequência: $1 \mathrm{~mL}$ de solução etanol 95\%, $5 \mathrm{~mL}$ de água destilada e $0,5 \mathrm{~mL}$ de reagente Folin Ciocalteau $1 \mathrm{~N}$, seguido de agitação. Após cinco minutos, foi adicionado $1 \mathrm{~mL}$ de solução de carbonato de sódio $5 \%(\mathrm{p} / \mathrm{v})$, seguindo para câmara escura por $60 \mathrm{~min}$. Transcorrido esse tempo, houve nova homogeneização e foi realizada leitura das absorbâncias no comprimento de onda 725 $\mathrm{nm}$. Os resultados foram expressos em mg ácido gálico equivalente (GAE)/g amostra úmida.

\section{Carotenoides totais}

A análise de carotenoides totais transcorreu de acordo com a metodologia de Lichtenthaler e Buschmann (2001) com adaptações. Para isso, transferiu-se 0,2 g do pó do resíduo da goiaba para os tubos de ensaio cobertos com papel alumínio. Em seguida, adicionou-se $18 \mathrm{~mL}$ de acetona $100 \%$ e colocou-se sob agitação por 30 segundos. Feito isso, a solução foi filtrada ao abrigo da luz com papel de filtro Whatman $n^{\circ} 1$, em seguida, foram 
realizadas leituras no espectrofotômetro nos seguintes comprimentos de onda $470 \mathrm{~nm}, 645 \mathrm{~nm}$ e $662 \mathrm{~nm}$. Os resultados foram expressos em $\mu \mathrm{g} / 100 \mathrm{~g}$ amostra úmida.

\section{Concentração de ácido ascórbico}

A determinação do teor de ácido ascórbico (AA), através do método titulométrico do 2,6-diclorofenol-indofenol, foi realizada conforme descrito por Oliveira et al. (2010) (método I). A solução titulante utilizada foi a solução de Tillmans. A solução titulada foi constituída de $1 \mathrm{~g}$ do pó e $50 \mathrm{~mL}$ de ácido metafosfórico $1 \%$, sob agitação por 2 minutos e em seguida filtrada em bomba à vácuo usando papel de filtro Whatman $n^{\circ} 1$. Em seguida, foi retirada alíquota de $10 \mathrm{~mL}$ dessa solução e titulada com a solução de Tillmans até surgir a coloração rosa, registrando o volume utilizado pela solução titulante. $\mathrm{O}$ teor de vitamina $\mathrm{C}$ foi expresso em $\mathrm{mg} / 100 \mathrm{~g}$ amostra úmida.

\section{Teste do radical 1,1 - Difenil-2-picrilhidrazil (DPPH)}

A avaliação da capacidade antioxidante utilizou o método do sequestro de radicais livres DPPH (2,2 difenil-1-picrilhidrazil) descrito por Duarte-Almeida et al. (2006). Em microplaca foi pipetado $40 \mu \mathrm{L}$ do extrato e $200 \mu \mathrm{L}$ de solução metanólica de DPPH. As amostras foram dispostas em câmara escura por 25 min e depois lidas em comprimento de onda de $517 \mathrm{~nm}$. Os resultados foram expressos em $\mu \mathrm{mol}$ Trolox equivalente (TE)/g amostra úmida.

\section{RESULTADOS E DISCUSSÃO}

Na Tabela 1 estão apresentados o teor de compostos fenólicos totais, carotenoides, ácido ascórbico e a atividade antioxidante de resíduo de goiaba vermelha desidratado em secador de bandejas.

Tabela 1 - Teor de compostos fenólicos totais, carotenoides, ácido ascórbico e atividade antioxidante do resíduo de goiaba vermelha desidratado.

\begin{tabular}{cccc}
\hline $\begin{array}{c}\text { Compostos fenólicos } \\
(\mathbf{m g} \text { GAE/100 g) }\end{array}$ & $\begin{array}{c}\text { Carotenoides } \\
(\boldsymbol{\mu g} / \mathbf{1 0 0} \mathbf{g})\end{array}$ & $\begin{array}{c}\text { Ácido ascórbico } \\
(\mathbf{m g} / \mathbf{1 0 0} \mathbf{~ g})\end{array}$ & $\begin{array}{c}\text { Atividade antioxidante } \\
(\mathbf{D P P H}-\boldsymbol{\mu m o l ~ T E} / \mathbf{g})\end{array}$ \\
\hline $133,73 \pm 7,03$ & $262,01 \pm 0,24$ & $47,58 \pm 0,04$ & $9,06 \pm 0,03$ \\
\hline
\end{tabular}

Valores referentes às médias média \pm desvio padrão $(\mathrm{N}=3)$.

Os compostos fenólicos fazem parte do metabolismo secundário dos vegetais, participando de maneira importante na defesa da planta. Esses compostos apresentam atividade antioxidante, característica apontada como relevante para o retardamento do envelhecimento e prevenção de doenças associadas ao estresse oxidativo (OLDONI, 2007; YAHIA, 2010).

O teor de compostos fenólicos totais determinado neste estudo foi superior ao relatado por Sousa et al. (2011b), ao analisarem extratos de resíduo de polpa de goiaba vermelha 
(24,63 mg GAE/100 g amostra úmida) utilizando o mesmo método. Essa diferença pode ser explicada pela concentração provocada pela retirada de água do produto durante a secagem. Entretanto, o valor obtido aqui foi inferior ao encontrado por Fu et al. (2011), ao analisarem extratos de polpa de goiaba vermelha (194,11 mg GAE/100 g amostra úmida).

Os carotenoides são importantes compostos bioativos amplamente encontrados na natureza, sendo responsáveis pela coloração das frutas e possuírem reconhecida atividade biológica. Várias frutas tropicais (açaí, buriti, goiaba, manga, abacaxi, acerola, mamão, caju, graviola, pitanga e sapoti) já foram apontadas como fonte de carotenoides (DEMBITSKY et al., 2011; PIERSON et al., 2012; SILVA et al., 2014). Em relação ao teor de carotenoides totais, neste estudo foi encontrado 262, $01 \mu \mathrm{g} / \mathrm{g}$ no resíduo desidratado. Em seu trabalho sobre frutas tropicais, Silva et al. (2014) apresentaram valor menor ao analisado aqui, no qual o teor de carotenoides totais para polpa de goiaba vermelha liofilizada correspondia a 26,67 $\mu \mathrm{g} / 100 \mathrm{~g}$ amostra. Porém, Sousa et al. (2011b) obteve resultado mais elevado em seu estudo sobre resíduo de goiaba vermelha in natura $(644,9 \mu \mathrm{g} / 100 \mathrm{~g}$ amostra).

É sabido que, a identificação de novas fontes de vitamina $\mathrm{C}$ na dieta é de grande interesse para a área de ciências da saúde, visto que é um componente essencial às funções fisiológicas do corpo (GÜÇLÜ et al., 2005). O teor de ácido ascórbico encontrado para o resíduo seco de goiaba vermelha é superior ao da polpa de outras frutas tropicais, conforme mostram Valente et al. (2011) e Almeida et al. (2011). Os autores encontraram teores de 12,2, 40,9, 3,1, 12,1, 13,0 e 3,3 mg/ $100 \mathrm{~g}$ amostra para polpas de maracujá, manga, tamarindo, umbu, abacaxi e pinha, respectivamente. No entanto, a amostra analisada neste trabalho apresentou teor de ácido ascórbico inferior ao encontrado por Sousa et al. (2011a) para resíduo de goiaba vermelha in natura $(75,90 \mathrm{mg} / 100 \mathrm{~g}$ amostra). Isso pode ser justificado pelo fato de que durante a secagem pode ter ocorrido perdas de ácido ascórbico, visto que esse composto é conhecido por ser um nutriente lábil, que pode ser degradado por um número de fatores, dentre eles $\mathrm{pH}$, teor de umidade, oxigênio, temperatura e presença de metais (KAYA et al., 2010).

Segundo Almeida et al. (2011), os alimentos ricos em antioxidantes desempenham um papel fundamental na prevenção de doenças. A capacidade antioxidante de diversas frutas varia de acordo com seu conteúdo de vitamina $\mathrm{C}$, carotenoides, bem como flavonoides e outros polifenóis (SAURA-CALIXTO E GONI, 2006; VON LINTIG, 2010). No que se refere ao método de redução do radical estável DPPH•, observa-se boa atividade antioxidante do extrato aquoso, o qual apresentou 9,06 $\mu \mathrm{mol}$ Trolox eq/g amostra. Este valor foi semelhante aos apresentados por Souza et al. (2012), para polpas de jenipapo (7,31 $\mu \mathrm{mol} \mathrm{TE} / \mathrm{g}$ amostra) e maracujá $(10,84 \mu \mathrm{mol}$ TE /g amostra). Prado (2009), ao analisar a capacidade antioxidante de polpa de goiaba vermelha, verificou valor inferior ao aqui apontado $(5,14 \mu \mathrm{mol} \mathrm{TE} / \mathrm{g}$ amostra). Por outro lado, Melo (2010) ao estudar a atividade antioxidante dos extratos de aquosos de resíduo de goiaba vermelha liofilizado, encontrou valor maior ao obtido neste trabalho $(16,28 \mu \mathrm{mol} \mathrm{TE} / \mathrm{g}$ amostra $)$.

\section{CONCLUSÃO}

Em resumo, este trabalhou mostrou que o resíduo de goiaba desidratado por convecção pode ser apontado como fonte promissora de compostos bioativos. Estudos 
complementares precisam ser conduzidos de forma a averiguar a aplicação desses produtos como ingredientes funcionais, na formulação de outros alimentos industrializados e diminuindo, dessa forma, a potencial contaminação ambiental causada pela excessiva quantidade produzida e incorreta disposição desses resíduos.

\section{REFERÊNCIAS}

ALMEIDA, M. M. B et al. Bioactive compounds and antioxidant activity of fresh exotic fruits from northeastern Brazil. Food Research International, v. 44, p. 2155-2159, 2011.

BRASIL. Ministério da Saúde. Secretaria de Políticas de Saúde. Coordenação-Geral da Política de Alimentação e Nutrição. Alimentos regionais brasileiros/ Ministério da Saúde, Secretaria de Políticas de Saúde, Coordenação-Geral da Política de Alimentação e Nutrição. - 1. ed. - Brasília: Ministério da Saúde, 2002.

CENTRO DE ESTUDOS AVANÇADOS EM ECONOMIA APLICADA - CEPEA. Valores do PIB do agronegócio brasileiro, 1994 a 2008. ESALQ-USP, 2010. Disponível em: <http://www.cepea.esalq.usp.br/pib/>. Acesso em: 20 abr. 2014.

CORREIA, R. T. P. et al. Production of phenolic antioxidants by the solid-state bioconversion pineapple waste mixed with soy flour using Rhizopus oligosporus. Process Biochemistry, v.39, p.2167- 2172, 2004.

CULTURA DA GOIABA. A Goiaba. Disponível em: <http://culturagoiaba.blogspot.com.br/2013/04/cultura-da-goiaba-fruticultura.html.>. Acesso em: 20 abr. 2014.

DEMBITSKY, V. et al. The multiple nutrition properties of some exotic fruits: biological activity and active metabolites. Food Research International, v. 44, p. 1671-1701, 2011.

DUARTE-ALMEIDA, J. M. et al. Avaliação da atividade antioxidante utilizando sistema bcaroteno/ácido linoléico e método de seqüestro de radicais DPPH. Ciência e Tecnologia de Alimentos, v.26, n.2, p.446-452, 2006.

FU, L. et al. Antioxidant capacities and total phenolic contents of 62 fruits. Food Chemistry, v.129, p. 345-350, 2011.

GÜÇLÜ, K et al. Spectrophotometric determination of ascorbic acid using copper (II)neocuproine reagent in beverages and pharmaceuticals, Talanta, v. 65, p.1226-1232,2005.

KAYA, A. et al. Drying conditions on the vitamin C (ascorbic acid) content of Hayward kiwifruits (Actinidia deliciosa Planch). Food and Bioproducts Processing, v. 8, n. 2, p. 165$173,2010$. 
LICHTENTHALER, H. K.; BUSCHMANN, C. Chlorophylls and carotenoids: Measurement and characterization by UV-VIS spectroscopy. Current protocols in food analytical chemistry, F4.3.1-F4.3.8, 2001.

MELO, P. S. Composição química e atividade biológica de residues agroindustriais. 2010, 100 f, Dissertação (Mestrado) - Universidade de São Paulo. Escola Superior de Agricultura Luiz Queiroz. Programa de Pós-Graduação em Ciência e Tecnologia de Alimentos. Piracicaba $-\mathrm{SP}$.

NASCIMENTO, R. J. Potencial antioxidante de resíduo Agroindustrial de goiaba. Universidade federal rural de Pernambuco. 2010, 110 f, Dissertação (Mestrado) Universidade Federal Rural de Pernambuco. Departamento de ciências domésticas. Programa de Pós-graduação em Ciência e Tecnologia de Alimentos. Recife - PE.

OLDONI, T. L. C. Isolamento e identificação de compostos com atividade antioxidante de uma nova variedade de própolis brasileira produzida por abelhas da espécie Apis mellifer. 2007. 154f. Dissertação (Mestrado em Ciência e Tecnologia de Alimentos) - Universidade de São Paulo. Piracicaba.

OLIVEIRA, R. et al. Otimização de metodologia colorimétrica para a determinação de ácido ascórbico em geléia de frutas. Ciência e Tecnologia de Alimentos, v. 30, n. 1, p. 244-249, 2010 .

PIERSON, J. T. et al. Major Australian tropical fruits biodiversity: bioactive compounds and their bioactivities. Molecular Nutrition \& Food Research, v. 56, n. 3, p. 357-387, 2012.

PRADO, A. Composição fenólica e atividade antioxidante de frutas tropicais. 2009, $100 \mathrm{f}$, Dissertação (Mestrado) - Universidade de São Paulo. Escola Superior de Agricultura Luiz Queiroz. Programa de Pós-Graduação em Ciência e Tecnologia de Alimentos. Piracicaba SP.

RAMOS, E. L. Desenvolvimento sustentável de sistemas de produção de frutas tropicais nativas e exóticas - abordagem mesoanalítica. Sitientibus. Feira de Santana/BA. n. 39, p.53$73,2008$.

ROBERTO, B. S. Resíduo de goiaba: metabolismo em ratos e Aplicabilidade em barras de cereais. 2012, 150 f, Dissertação (Mestrado) - Universidade Federal de Santa Maria. Centro de Ciências Rurais. Programa de Pós-Graduação em Ciência e Tecnologia dos Alimentos. Santa Maria - RS.

SAURA-CALIXTO, F.; GONI, I. Antioxidant capacity of the Spanish Mediterranean diet. Food Chemistry, v. 94, n.3, 442-447, 2006.

SILVA, L. M. R. et al. Quantification of bioactive compounds in pulps and by-products of tropical fruits from Brazil. Food Chemistry, v. 143, n. 15, p. 398-404, 2014. 
SOUSA, M. S. B. et al. Caracterização nutricional e compostos antioxidantes em resíduos de polpas de frutas tropicais. Ciênc. agrotec., v. 35, n. 3, p. 554-559, $2011 \mathrm{a}$.

SOUSA, M. S. B. et al. Fenólicos totais e capacidade antioxidante in vitro de resíduos de polpas de frutas tropicais. Braz. J. Food Technol., Campinas, v. 14, n. 3, p. 202-210, 2011 b.

SOUZA, V. R. et al. Determination of bioactive compounds, antioxidant activity and chemical composition of Cerrado Brazilian fruits. Food Chemistry, v. 134, p. 381-386, 2012.

TOLENTINO, V. R.; SILVA, A. G. Processamento de vegetais: frutas/polpa congelada. Programa Rio Rural. Manual Técnico; 12. Niterói: Programa Rio Rural, 2008.

VALENTE, A. et al. Ascorbic acid content in exotic fruits: A contribution to produce quality data for food composition databases, Food Research International, v. 44, n. 7, p. 2237-2243, 2011.

VON LINTIG, J. Colors with functions: Elucidating the biochemical and molecular basis of carotenoid metabolism. Annual Review of Nutrition, v. 30, p. 35-56, 2010.

YAHIA, E. M. The Contribution of Fruit and Vegetable Consumption to Human Health. In: ROSA, L.A.; ALVAREZ-PARRILLA, E.; GONZALEZ-AGUILARA; G.A. Fruit and vegetable phytochemicals: chemistry, nutritional value and stability. Hoboken: WileyBlackwell, 2010. p. 3-51. 